\title{
DUPLEX METHYLATION-SPECIFIC SEMI-QUANTITATIVE REAL-TIME PCR FOR COST-EFFECTIVE \& TIME-EFFICIENT DIAGNOSTIC SCREENING OF CHROMOSOME 15 AND 14 IMPRINTED REGIONS
}

\author{
Ruszova, $\mathbf{E}^{1}$, Chmelarova $\mathbf{M}^{2}$, Senkerikova $\mathbf{M}^{1}$, Stefackova $\mathbf{S}^{3}$ \\ ${ }^{1}$ Department of Clinical Genetics, University Hospital in Hradec Kralove, ${ }^{2}$ Institute for Clinical Biochemistry and \\ Diagnostics, University Hospital in Hradec Kralove, ${ }^{3}$ Department of Pediatric Neurology, University Hospital in \\ Hradec Kralove, Czech Republic
}

\begin{abstract}
Purpose: Our goal was to develop two-tier strategy based on in house-designed methylation specific-duplex polymerase chain reactions (MS-PCRs) that could serve as a relatively simple, cost effective, time efficient approach for molecular screening of imprinted regions on chromosomes 15 and 14.

Patients and methods: Patients were referred to examination during infancy due to hypotonia and motor development delay. Duplex MS-PCRs were performed that enabled detection of methylated/unmethylated DNA in NDN and MEG3 CpG islands via plurality of detection channels on PCR instrument Rotor Gene 6000.

Results and discussion: Both, copy number variations as well as methylation changes, were revealed by our in house-designed methodology by focusing $N D N$ gene. No imprinting aberrations were yet discovered in MEG3 gene. Clinical features of the patients were compared. In agree with literature no typical facial features were observed in PWS patient with imprinting defect and AS UPD patient seems to have a relatively better development and language ability in comparison to deletional form of the disease.

Conclusion: In conclusion we were able to establish new, throughput and robust diagnostic approach to PWS/AS.
\end{abstract}

Keywords: Prader Willi syndrome, Angelman syndrome, methyl-specific semi-quantitative real-time PCR, epigenetics

$\begin{array}{ll}\text { Abbreviations: } & \\ \text { AS } & \begin{array}{l}\text { Angelman syndrome } \\ \text { differentially methylated region }\end{array} \\ \text { DMR } & \text { maternally expressed 3 (non-protein coding), aliases GTL2 } \\ \text { MEG3 } & \text { methylation-specific polymerase chain reaction } \\ \text { MS-PCR } & \text { necdin, melanoma antigen (MAGE) family member } \\ \text { NDN } & \text { Prader Willi syndrome } \\ \text { PWS } & \text { uniparental disomy } \\ \text { UPD } & \text { maternal uniparental disomy for chromosome 14 } \\ \text { [upd(14)mat] } & \text { paternal uniparental disomy for chromosome 14 }\end{array}$

\section{INTRODUCTION}

Central hypotonia is one of the most difficult issues in neurology, and ruling out neurogenetic syndrome causes seems to be crucial for establishing the correct molecular diagnosis and clinical prognosis. Several studies have addressed the relative frequency of the various causes of neonatal/infantile hypotonia $(1,2)$. Uniparental disomy (UPD) of chromosomes 15 and 14 gives rise to two genetic conditions which are particularly important in relation to this phenomenon. UPD results in imbalance of the expression of imprinted

Address for correspondence:

Mgr. Ema Ruszova, PhD.,Dept. of Clinical Genetics, University Hospital, Sokolska 581, 50005 Hradec Kralove, Czech Republic; Tel +420 495833 348; E- mail: ema.ruszova@fnhk.cz 
genes and leads to abnormal development (3). Prader Willi (PWS) [OMIM (Online Mendelian Inheritance in Man) No. 176270] and Angelman syndrome (AS) [OMIM No. 105830] are clinically distinct neurological disorders caused by a lack of expression of oppositely imprinted genes in chromosome region 15q11-q13; PWS is caused by loss of expression from genes on the paternally inherited allele (MKRN3, MAGEL2, NDN, PWRN1, C15orf2, SNURF-SNRPN and several snoRNA genes) (4), and the absence of the maternal copy of the same region causes AS (5). The population prevalence is estimated at 1:10 000-1:16 000 (6).

Both syndromes have their own clinical picture which varies at different ages. Prader Willi syndrome is the cause of extreme childhood obesity, which leads to serious morbidity and early mortality (7). The main features of AS are intellectual disability, lack of speech, seizures, and a characteristic behavioral profile. The behavioral features of AS include a happy demeanor, easily provoked laughter, short attention span, hypermotoric behavior, mouthing of objects, sleep disturbance, and an affinity for water (8).

In addition, both types of uniparental disomy can be recognised also on chromosome 14: [upd(14)mat] and [upd(14)pat], the latter associated with a more severe phenotype as compared to upd(14)mat (9). Mitter and his colleagues recently reported that upd(14)mat was detected in patients who were suspected of having PWS, and raised the question that upd(14)mat could be present in patients with PWS-like phenotype (3). The imprinting cluster at chromosome 14q32 involves differentially methylated region at the MEG-3 gene (MEG3-DMR) (10, 11).

Although greater than 99\% of PWS is associated with a detectable methylation abnormality in the imprinted region, this only applies to $78 \%$ of AS (12), and therefore the inability to assess other mechanisms resulting in AS may be a limitation of the epigenetic approach (13).

In this strategy, integrating duplex semi-quantitative real-time PCR amplification of bisulfite-modified DNA was performed to detect the maternally and paternally imprinted NDN gene. After exclusion of a PWS/AS diagnosis, suspected patients who presented with a PWSlike phenotype underwent DNA methylation analysis of the MEG3 gene. Interestingly, these two sequential methodology steps allow a rational, simple and robust diagnostic approach to epigenetic-based hypotonia in infancy.

\section{MATERIAL AND METHODS}

\section{DNA isolation}

This study was approved by the Ethical Review Board of the University Hospital, Hradec Kralove, Czech Republic. All legal guardians of patients provided written informed consent and received genetic counselling. DNA was extracted from peripheral blood using the QIAamp DNA Blood Midi Kit (Qiagen, Hilden, Germany) according to the manufacturer's instructions with no modifications. The purity and DNA concentration was determined by NanoDrop ${ }^{\circledR}$ ND-1000 UV-Vis Spectrophotometer.

\section{Bisulfite DNA Modification}

The genomic DNA (500 ng) was modified by sodium bisulfite treatment using the EZ DNA Methylation-Gold Kit (D5005, ZymoResearch, Irvine, CA, USA) according to the manufacturer's instructions.

\section{Design of MethyLight Probes and Methylation-Specific PCR}

MethyLight Assays enable discrimination between unmethylated and methylated sites in the same sample. The FAM-labeled TaqMan probe always detects the methylated site, whereas the HEX-labeled TaqMan probe always detects the unmethylated site in our protocol (Fig. 2). MethyLight probes and primers were designed using the Beacon Designer ${ }^{\mathrm{TM}}$ v.8 (Premier Biosoft International). Sequence data for the NDN and MEG3 gene were obtained 
from the GeneBank database (accession number NG_009380.1 and NG_016853.1 respectively). The oligonucleotides used in this study are described in Tab. 1.

MethyLight fluorescent probes and primers were synthesized by Generi Biotech s.r.o. (Hradec Kralove, Czech Republic) and were used at concentrations of $300 \mathrm{nM}$ and $900 \mathrm{nM}$, respectively (Fig. 1).

Table 1: Oligonucleotides used in the study

\begin{tabular}{|l|l|}
\hline NDN gene & Ref.seq. NG_009380.1 \\
\hline NDN-F-Un & GGTGAAAGATGTTATTGGTAGTTATAAGAA \\
\hline NDN-R-Un & CCAACACAAACTCCATAATATACAAACT \\
\hline NDN-F-Me & GTGAAAGATGTTATCGGTAGTTATAAGAAG \\
\hline NDN-R-Me & CAACGCAAACTCCATAATATACAAACT \\
\hline P-Me-NDN-Fam & CTCAAATACAACCCGAACACCCGAACGAA \\
\hline P-Un-NDN-Hex & ACCTCAAATACAACCCAAACACCCAAACAA \\
\hline MEG3 gene & Ref.seq. NG_016853.1 \\
\hline MEG3-Me-F & GGATTTATTGGAGGATTTTGGATTCG \\
\hline MEG3-Un-F & GATTTATTGGAGGATTTTGGATTTGT \\
\hline MEG3-Me-R & AATCAACTAACGTACCTCTATACGC \\
\hline MEG3-Un-R & AAAATCAACTAACATACCTCTATACAC \\
\hline P-Me-MEG3-Fam & TCCACAACACCCGAAACCACGACG \\
\hline P-Un-MEG3-Hex & TCCACAACACCCAAAACCACAACACAA \\
\hline
\end{tabular}

Abbreviations: Un - for unmethylated DNA, Me- for methylated DNA, P-probe, F-forward, R-reverse

Methylation-specific PCR (MS-PCR) was done using 21 of bisulfite-modified DNA as a template. Bisulfite-modified DNA was amplified in a $25 \mu \mathrm{L}$ reaction volume with the primer/probe sets for duplex PCR and Platinum ${ }^{\circledR}$ Taq PCRx DNA polymerase (Invitrogen ${ }^{\mathrm{TM}}$ Life Technologies, Karlsrube, Germany). Samples were heated to $95^{\circ} \mathrm{C}$ for 3 min and then amplified for 45 cycles consisting of $95^{\circ} \mathrm{C}$ for $25 \mathrm{~s}, 50^{\circ} \mathrm{C}$ for $40 \mathrm{~s}$, and $69^{\circ} \mathrm{C}$ for $20 \mathrm{~s}$, followed by a final extension step at $72^{\circ} \mathrm{C}$ for $10 \mathrm{~min}$ (Rotor Gene 6000, Corbett Research, Australia). Fluorescence was acquired in the green and yellow channel of the instrument in a single reaction. MS-PCRs were validated using commercial DNA controls (Human Methylated \& Non-methylated DNA set, cat.n. D5014, ZymoResearch, Irvine, CA, USA) and DNA from patients whose diagnoses were confirmed using widely conducted commercial assay, multiplex ligation-dependent probe amplification (MLPA, ME028, MRC Holland) and fluorescence in situ hybridization (deletional forms). 
Chromosome 15: 23,685,391-23,687,326

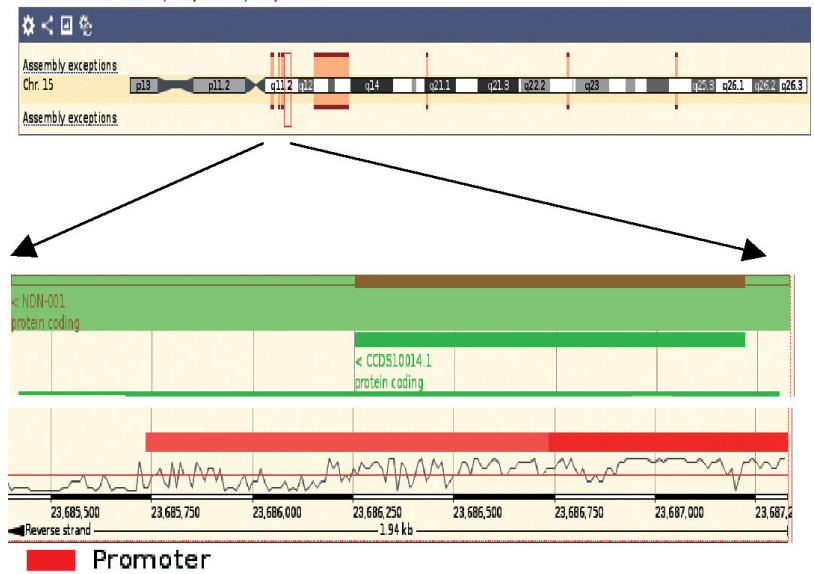

Chromosome 14: 100,779,410-100,861,031

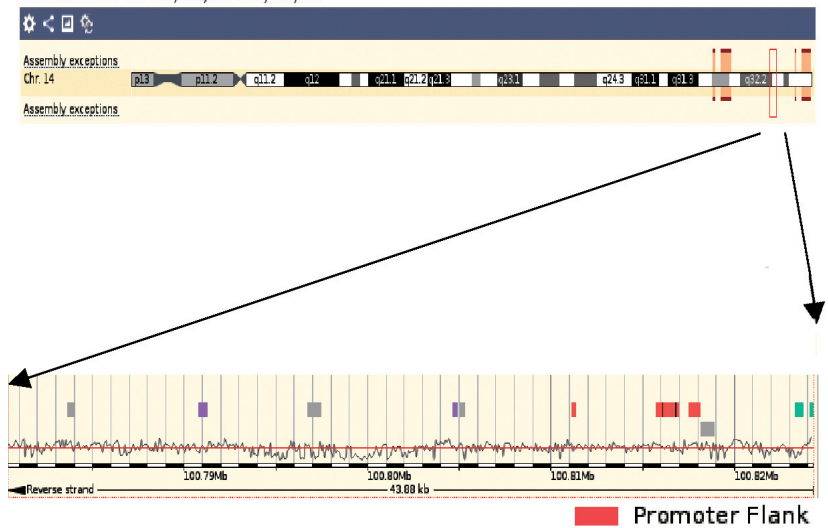

NG_009380.1, CpG island 4295 -6294bp NG_016853.1, CpG island 1-2000bp (predicted via Methyl Primer Express ${ }^{\circledR}$ v. 1.0, Applied Biosystems) Product length-129bp (5459-5587bp) Product length-151bp (1284-1435bp) yellow colour- CpG sites pink colour- non-CpG sites ( C" " converted to"T" by PCR)

\section{Methylated DNA}

\section{GTGAAAGATGTTATCGGTAGTTATAAGAAG}

TGGTGTAGGAGTATTTTCGGCGTATTAGTTTATTTTCG TTCGGGTGTTCGGGTTGTATTGAG GTTAATT

AGTTTGTATA TTATGGAGTTTGCGTTG

\section{Unmethylated DNA}

GGTGAAAGATGTTATTGGTAGTTATAAGAA

GTGGTGTAGGAGTATTTTTTGGTGTATTAGTTTTATTT TTGTTTEGGTGTTTGGGTTGTATTTGAGGT TAATT ÄTTTGTATATTATGGAGTTTG TGTTG

\section{Methylated DNA}

GGATTTATTGGAGGATTTTGGATTCG

TTTATTTAAGAGGGAATAGTTTTGAGATTTTCGGA TTTATGCGGAGTGGTCGTAGAGGTCGGGGTCGGTT TTTG CGTCGTGGTTTCGGGTGTTGTGGA G GCGTAT $\underline{A}$ AAGGTA

\section{Unmethylated DNA}

GGATTTATGGAGGATTTGGATTTGT

TTATTAAGAGGGAATAGTTTGAGATTTTMGATT ATT TTGGAGTGGTGTAGAGGTTGGGGTTGGTT TTGT TTGGGTTTIGGTGTTGTGGA GTG

Fig. 1 Genes, CpG island prediction, CpG sites in oligonucleotides 


\section{Determination of methylation level}

To assign a semi-quantitative measurement of the DNA methylation level, a methylation index was calculated for each sample using the formula:

$$
\mathrm{MI} \%=\left(100 /\left[1+2^{\mathrm{Ctme}-\mathrm{Ctun}}\right]\right)
$$

where MI is the methylation index as previously described (14), Ctun is the average cycle threshold $(\mathrm{Ct})$ obtained from duplicate quantitative PCRs using the unmethylated primer pair, and Ctme is the average Ct obtained using the methylated primer pair.

\section{RESULTS}

We identified abnormal hypermethylation in the NDN gene in 2 patients from 24 with central hypotonia. In one case a small deletion involving the NDN gene was present and in the other an imprinting defect was detected. Abnormal hypomethylation was found at the same gene in the same ratio (2/24), again one with a small deletion and one with upd(15)pat. Since NDN DMR which was used for the diagnostic methylation test is involved in the shortest region of deletion overlap, this combined analysis is suitable for detecting both copy number aberrations as well as methylation changes. Nevertheless we did not find any [upd(14)mat] or [upd(14)pat].

\section{CASE1 (PWS-deletional form)}

The child was presented for genetic examination at the age of three years. There had been decreased fetal movement during pregnancy. Central hypotonia, feeding difficulties and distinctive facial features were reported in early infancy. However in later infancy obesity developed due to excessive eating, there was motor developmental delay (walking not until 20 months) and delayed speech, and short stature was observed (at age 3y her weight was 20kg). The deletional form of PWS was established in this case. Growth hormone therapy was applied from 3y10m, but excessive obesity persisted (at age 5y4m her weight was 30kg).

\section{CASE2 (PWS-imprinting defect)}

This newborn was examined for central hypotonia, with decreased newborn reflexes and testis retention. Fetal movements were decreased during pregnancy also. An abnormal methylation pattern was found, but deletion and upd(15)mat were excluded. Further clinical monitoring was modified with growth hormone therapy (started form the age of 5 months) and strict supervision of daily food intake. At $14 \mathrm{~m}$ his weight was $9,5 \mathrm{~kg}$ and height $80 \mathrm{~cm}$ but mild delayed motor development was evident at that time.

\section{CASE3 (AS-deletional form)}

A $21 \mathrm{~m}$-old boy had suffered perinatal asphyxia due to umbilical cord strangulation, but needed only tactile stimulation to initiate spontaneous respiration after birth. His early postnatal adaptation occurred without complication. Later, severe developmental delay, absence of speech, seizures and tremor of the body, and borderline microcephaly appeared. The deletional form of AS was proven in this child.

\section{CASE4 (AS-UPD)}

An 8y-old boy presented with developmental delay (walking not until 25m), borderline microcephaly, epileptic seizures from 7y, serious speech failure (use of only six words), sleep disturbance, hypermotoric behaviour, and frequent laughter. Upd(15)pat for a critical region of chromosome 15 was detected. 


\section{DISCUSSION}

Epigenetics, as broadly defined, is the study of heritable changes in gene expression that are not caused by changes in the primary DNA sequence (15). Epigenetic dysregulation of the imprintome is the cause of specific genetic syndromes, which show alterations in growth, metabolism and neurologic development (16). In particular, early studies identified epigenetic-based aberrations that are responsible for causation of diseases, such as Prader Willi and Angelman syndrome, both accompanied by infancy hypotonia. Investigation of methylation changes are requested every day by the genetic counsellors. According to the guidelines (4), the recommended strategy for analysis of PWS and AS is methylation patterntracking within 15q11-15q13 using molecular genetic techniques that determine copy number changes (deletional type) and methylation changes in a single tube. The promoter methylation status of the NDN gene is recognized as a valid diagnostic characteristic (17). There are many commercial assays on the market, but the cost of such kits is a disadvantage. Our goal was to develop our own real-time duplex MS-PCR methodology, which would serve as a robust and cost effective pre-screening process.

As the MethyLight probes are designed to contain CpG sites in both probes as well in the primers (Fig. 1), they do not inspire much confidence among Czech molecular biologists.

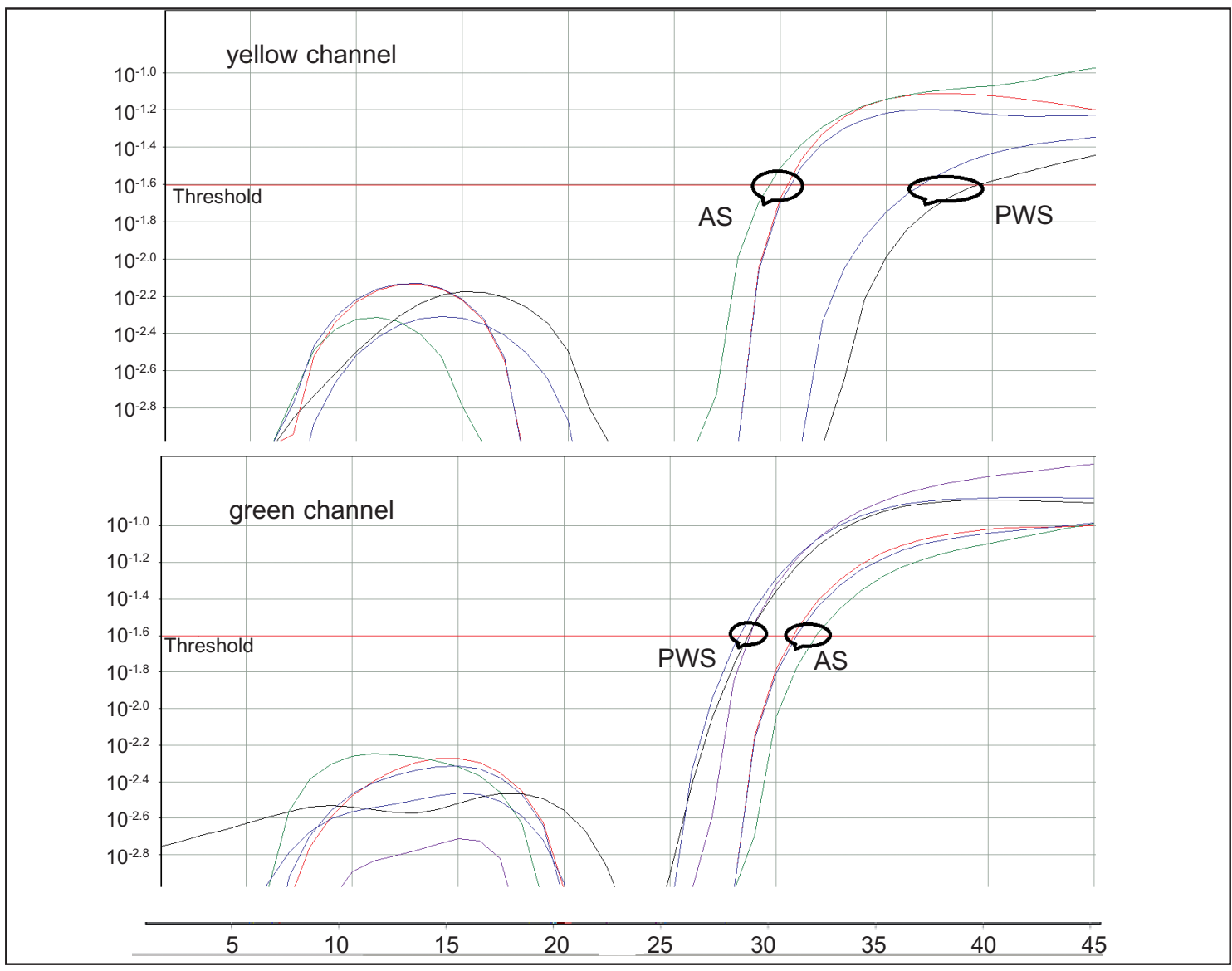

Fig. 2 Detection of unmethylated DNA in the yellow channel and methylated DNA in the green channel of RG6000 real-time PCR instrument (Corbett Research) 
Nevertheless we decided to take a chance and designed two MethyLight assays for 2 genes: NDN and MEG3. Thermocyclers with a plurality of detection channels then allow the formation of multiplexing type reaction which can simultaneously detect both the methylated and unmethylated DNA in a single reaction (Fig. 2). In addition, it is a high-throughput analysis and as an alternative technique for semi-quantitative analysis of target genes, and the avoidance potential contamination, is more robust. Moreover, MS-PCR can be performed without the need for parental DNA testing. During validation of the methodology, there appeared only one risk factor, and this was the background fluorescent signal from the second channel. Hence the presence of positive control DNAs seemed to be a prerequisite for good evaluation of MI tresholds, drawing attention to aberrant methylation.

No methylation aberration was discovered in MEG3 gene. We may speculate that the number of examined patients was too small (24) to find any upd(14).

Patients were referred to us during infancy due to hypotonia and motor development delay. Clinical features of these patients with deletional forms were compared with those with other forms of the disease. In consensus with the literature $(18,19)$ no typical facial features were observed in PWS patient with imprinting defect. In both cases of PWS some degree of cognitive impairment was reported. Early diagnosis with hormonal therapy starting at a very young age, and early onset strict supervision of daily intake in CASE2 has led to significant benefit.

All genetic mechanisms in AS lead to severe-to-profound intellectual disability, movement disorder, characteristic behaviors, and severe limitations in speech and language. The deletional form results in the most severe phenotype with microcephaly, seizures, motor difficulties and language impairment, compared to individuals with UPD or imprinting defects. Individuals with UPD have better physical growth (e.g. less likelihood of microcephaly), fewer movement abnormalities, and a lower prevalence of seizures than do those with other underlying molecular mechanisms (20-22).

Assessment of the disease etiology is crucial for determining the prognosis of the neurodevelopmental severely hypotonic child, which in the case of Prader Willi syndrome is very favorable. Early diagnosis, established cooperation with the family, a suitable diet, and treatment with reference to the specificities of this syndrome brings substantial benefits for both the patient and his family, and for society. Even death in childhood is not exceptional due to late detection and lack of cooperation from the family on a suitable diet regime and treatment. It seems to be equally important from our experiences to investigate PWS also in the case of severe muscle hypotonia during the neonatal period. PWS is also considered for differential diagnosis in all cases of significant childhood obesity.

An equally important issue in families with PWS/AS is the prognosis and the chance of occurrence of the disease elsewhere in the family. The risk of recurrence can only be established after knowing the exact genetic etiology, and it may vary according to the type from unimportant (UPD, deletion) to a 50\% risk (an imprinting defect)(19).

\section{CONCLUSIONS}

The purpose of this paper is to report an MS-PCR-based assay evaluating the CpG islands in NDN and MEG3 genes as routine molecular screening justified for patients suspected of having PWS/AS. We managed to create a two-tier strategy for the differential diagnosis of PWS, where, after exclusion of methylation abnormalities in the chromosome 15 we continued with the exclusion of a PWS-like phenotype linked to chromosome 14. 


\section{REFERENCES}

1. Paro-Panjan D, Neubauer D. Congenital hypotonia: is there an algorithm? J Child Neurol. 2004; 19(6):439-42.

2. Leyenaar J, Camfield P, Camfield C. A schematic approach to hypotonia in infancy. Paediatr Child Health. 2005; 10(7):397-400.

3. Mitter D, Buiting K, von Eggeling F, Kuechler A, Liehr T, Mau-Holzmann UA, et al. Is there a higher incidence of maternal uniparental disomy 14 [upd(14)mat]? Detection of 10 new patients by methylation-specific PCR. Am J Med Genet A. 2006;140(19):2039-49.

4. Ramsden SC, Clayton-Smith J, Birch R, Buiting K. Practice guidelines for the molecular analysis of Prader-Willi and Angelman syndromes. BMC Med Genet. 2010;11:70.

5. Askree SH, Dharamrup S, Hjelm LN, Coffee B. Parent-of-origin testing for 15q11-q13 gains by quantitative DNA methylation analysis. J Mol Diagn. 2012;14(3):192-8.

6. Burman P, Ritzen EM, Lindgren AC. Endocrine dysfunction in Prader-Willi syndrome: a review with special reference to GH. Endocr Rev. 2001;22(6):787-99.

7. Butler MG. Prader-Willi Syndrome: Obesity due to Genomic Imprinting. Current Genomics. 2011;12(3):204-15.

8. Bird LM. Angelman syndrome: review of clinical and molecular aspects. Appl Clin Genet. 2014;7:93-104.

9. Murphy SK, Wylie AA, Coveler KJ, Cotter PD, Papenhausen PR, Sutton VR, et al. Epigenetic detection of human chromosome 14 uniparental disomy. Hum Mutat. 2003;22(1):92-7.

10. Irving MD, Buiting K, Kanber D, Donaghue C, Schulz R, Offiah A, et al. Segmental paternal uniparental disomy (patUPD) of 14q32 with abnormal methylation elicits the characteristic features of complete patUPD14. Am J Med Genet A. 2010;152A(8):1942-50.

11. Hosoki K, Kagami M, Tanaka T, Kubota M, Kurosawa K, Kato M, et al. Maternal uniparental disomy 14 syndrome demonstrates prader-willi syndrome-like phenotype. J Pediatr. 2009; 155(6):9003 el.

12. Wang HL, Liang DS, Xia Y, Xia C, Wu LQ. [Genetic diagnosis and prenatal diagnosis of Angelman syndrome]. Zhonghua Yi Xue Yi Chuan Xue Za Zhi. 2009;26(5):511-3.

13. Wang W, Law HY, Chong SS. Detection and discrimination between deletional and non-deletional Prader-Willi and Angelman syndromes by methylation-specific PCR and quantitative melting curve analysis. J Mol Diagn. 2009;11(5):446-9.

14. Fu A, Leaderer D, Zheng T, Hoffman AE, Stevens RG, Zhu Y. Genetic and epigenetic associations of circadian gene TIMELESS and breast cancer risk. Mol Carcinog. 2012;51(12):923-9.

15. Rodenhiser D, Mann M. Epigenetics and human disease: translating basic biology into clinical applications. CMAJ. 2006;174(3):341-8.

16. Skaar DA, Li Y, Bernal AJ, Hoyo C, Murphy SK, Jirtle RL. The human imprintome: regulatory mechanisms, methods of ascertainment, and roles in disease susceptibility. ILAR J. 2013;53(34):341-58.

17. Jay P, Rougeulle C, Massacrier A, Moncla A, Mattei MG, Malzac P, et al. The human necdin gene, NDN, is maternally imprinted and located in the Prader-Willi syndrome chromosomal region. Nat Genet. 1997;17(3):357-61.

18. Wey E, Bartholdi D, Riegel M, Nazlican H, Horsthemke B, Schinzel A, et al. Mosaic imprinting defect in a patient with an almost typical expression of the Prader-Willi syndrome. Eur J Hum Genet. 2005; 13(3):273-7.

19. Cassidy SB, Dykens E, Williams CA. Prader-Willi and Angelman syndromes: sister imprinted disorders. Am J Med Genet. 2000;97(2):136-46.

20. Valente KD, Varela MC, Koiffmann CP, Andrade JQ, Grossmann R, Kok F, et al. Angelman syndrome caused by deletion: a genotype-phenotype correlation determined by breakpoint. Epilepsy Res. 2013;105(1-2):234-9.

21. Lossie AC, Whitney MM, Amidon D, Dong HJ, Chen P, Theriaque D, et al. Distinct phenotypes distinguish the molecular classes of Angelman syndrome. J Med Genet. 2001;38(12):834-45.

22. Saitoh S, Wada T, Okajima M, Takano K, Sudo A, Niikawa N. Uniparental disomy and imprinting defects in Japanese patients with Angelman syndrome. Brain Dev. 2005;27(5):389-91.

Acknowledgment: The study was supported by the project (Ministry of Health, Czech Republic) for conceptual development of research organization 00179906.

Received: September, 8, 2015

Accepted: October, 20, 2015 